\title{
De los políticos a la universidad: análisis del debate sobre el cierre de \#RTVV en Twitter
}

\section{Politikariak eta unibertsitatea: \#RTVV ixteari buruz Twitterren egindako eztabaidak aztergai}

\section{From politicians to university: an analysis of the debate about the closing of \#RTVV on Twitter}

\section{Fátima Ramos del Cano ${ }^{1}$ Sonia González Molina²}

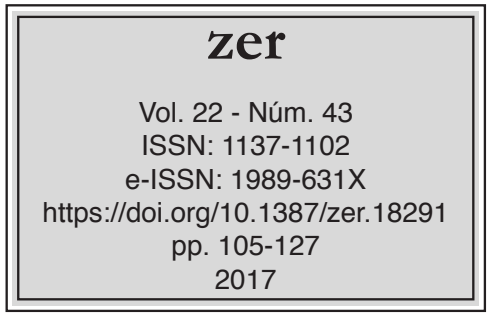

Recibido el 10 de octubre de 2016, aceptado el 12 de julio de 2017.

\begin{abstract}
Resumen
El artículo analiza el discurso en Twitter de los principales actores implicados en el cierre de la radiotelevisión pública valenciana (RTVV). La metodología se basa en el análisis de contenido de 1.014 tuits y 904 hashtags publicados por políticos, periodistas afectados, medios y universidades públicas valencianas para determinar los temas y funciones de encuadre que emplearon en sus mensajes sobre el proceso. El análisis indica que los contenidos que más se publican son de tipo informativo y que cada actor privilegia un encuadre de acuerdo con sus intereses.
\end{abstract}

Palabras Clave: RTVV, redes sociales, radiotelevisión, discurso, encuadre.

\section{Laburpena}

Valentziako Erkidegoko irrati-telebista publikoaren (RTVV) ixtean inplikatutako eragile nagusiek Twitterren argitara eman duten diskurtsoa aztergai du artikuluak. Valentziako Erkidegoko hainbat politikari, kazetari kaltetu, komunikabide eta unibertsitate publikok argitaratutako 1.014 txioren eta 904 hashtagen edukien azterketan oinarritu da metodologia, prozesuari buruzko mezuetan erabilitako gaiak eta ikuspegiak zehazte aldera. Analisiak adierazi du gehien argitaratutako edukiak informatzaileak direla, eta eragile bakoitzak ikuspegi jakin bati eman diola lehentasuna, norberaren interesen arabera.

Gako-hitzak: RTVV, sare sozialak, irrati eta telebista, diskurtsoa, enkoadraketa.

\footnotetext{
Universitat Jaume I (Castellón), framos@uji.es

2 Universitat Jaume I (Castellón), smolina@uji.es
} 


\begin{abstract}
The article analyses the discourse on Twitter of the main actors involved in the closing of "Ràdio Televisió Pública Valenciana" (RTVV). The methodology is based on the content analysis of 1,014 tweets and 904 hashtags published by politicians, journalists, media and Valencian public universities. The aim is to determine the themes and framing functions that they used in their messages about this process. The results show that the most published contents are the informative ones and that each actor seeks to privilege a frame according to their interests.
\end{abstract}

Keywords: RTVV, social networks, radio and television, discourse, framing. 


\section{Introducción: la crisis del modelo de las radiotelevisiones públicas}

El 5 de noviembre de 2013 el Gobierno Valenciano anunció la suspensión ${ }^{3}$ de las emisiones y el cierre de Ràdio Televisió Valenciana (RTVV), lo que supone un punto de inflexión en la historia de la radiotelevisión pública española. Desde 1983, momento en el que comienzan las primeras emisiones de la Radio Televisión Vasca Euskal Irrati Telebista (ETB), España había desarrollado un sistema audiovisual dependiente del Estado excepcional en el marco europeo (Miguel de Bustos y Casado, 2015). Así, hasta el cierre de ente público valenciano, 13 de las 17 comunidades autónomas contaban con su propio servicio público autonómico radiotelevisivo.

La academia defiende la permanencia de las televisiones públicas por su capacidad para vincularse con valores como los de la identidad cultural, la diversidad programática o el pluralismo ideológico (García Santamaría y Pérez Serrano, 2015). Su presencia, además, también se considera como motor esencial del desarrollo económico, social y cultural de la industria en su radio de acción, ya sea nacional, local o regional (Soler Campillo y Marzal Felici, 2015).

Autores como Albornoz y Cañedo (2015) sitúan en 1999 el comienzo de la crisis del modelo antes expuesto. La escasa rentabilidad económica, la exigua justificación de la subvención pública (Miguel de Bustos y Casado, 2012), así como las malas prácticas informativas y la excesiva politización de sus consejos de administración (Fernández Lombao y Campos Freire, 2013) son algunos de los principales problemas a los que aún hoy en día se enfrenta la televisión pública. La presión comercial creciente y la dificultad de combinar una oferta masiva con los valores propios del servicio público son sus principales desafíos (Benson, y Powers, 2011).

En este contexto, la Comunidad Valenciana se convierte en la primera en ver disuelta su Corporación de Radio Televisión el 29 de noviembre de 2013, cosa que generó una intensa controversia. Este artículo se propone analizar el discurso en la red social Twitter de gobierno, oposición, medios de comunicación, trabajadores de RTVV y universidades públicas valencianas con estudios de Comunicación. Son colectivos implicados en la decisión de cierre o afectados por la misma. Al tratarse de un caso sin precedentes, esta investigación es una oportunidad para examinar los temas y estrategias discursivas empleadas por los medios e instituciones involucrados en el debate social que surgió al respecto en las redes.

\section{Las redes sociales y la democratización del discurso mediático}

La llegada de Internet y, sobre todo, de las redes sociales, ha permitido crear un escenario comunicativo más competitivo (Chadwick, 2011) al descomponerse el monopolio que hasta ahora las élites políticas y los profesionales de la información tenían con respecto a la construcción de la realidad social y política (McNair, 2006). Las plataformas propias de la Web 2.0 permiten a los ciudadanos y colectivos sociales ostentar el papel de productores y consumidores y lograr tener voz (Casero-Ripollés y Feenstra, 2012) en un proceso comunicativo que se torna más democrático (Castells, 2009) y transparente (Della Porta, 2011).

Véase: http://www.lasprovincias.es/apoyos/documentos/Comunicado-cierre-RTVV.pdf 
Según Toret (2013), nos encontramos en un contexto de apropiación masiva de las herramientas digitales para la acción colectiva y el ejercicio del escrutinio público y el contrapoder (Casero-Ripollés y Feenstra, 2012). La ciudadanía activa tiene en su mano la posibilidad de transformarse en una comunidad "vigilante de la libertad de expresión" atenta a las posibles amenazas de los gobiernos (Castells, 2014: 8-9). En este contexto, las redes sociales pueden generar nuevas formas de acción colectiva e incrementar la eficiencia en la difusión pública de actividades y acciones de resistencia ciudadanas (Van Laer y Van Aelst, 2010; Bennet y Segerberg, 2012). El trabajo de Valenzuela, Park y Kee (2009) ya ponía de manifiesto la relación directa entre el uso de las redes sociales y el compromiso con acciones cívicas y/o políticas.

La dinámica de distribución de contenidos a través de las redes responde al denominado momento de "mayor trauma colectivo". Aquí, plataformas como Twitter ejercen un efectivo mecanismo de sensibilización ciudadana y movilización (Berná, Pérez y Arroyas, 2013) al actuar como corrientes de conciencia social (Naaman, Becker y Gravano, 2011) y programar y estimular la conversación digital en las estrategias de comunicación de partidos políticos emergentes (Casero-Ripollés, Feenstra y Tormey, 2016).

La literatura ha estudiado las redes sociales, en especial Twitter, desde el punto de vista de las motivaciones y rutinas de empleo por parte de los usuarios (Huberman et al., 2009), de la dinámica y patrones que utilizan con el propósito de difundir información (Wu et al., 2011), o incluso como plataformas de periodismo alternativo a las tradicionales, especialmente por parte de colectivos de activistas que las emplean como mecanismo para comunicar sus puntos de vista. Ejemplo de ello son las protestas contra la cumbre del G-20 en Toronto (Poell y Borra, 2012) o el movimiento de los indignados durante el 15-M en España (Micó y Casero-Ripollés, 2013).

Asimismo, investigaciones precedentes también han puesto de manifiesto algunos riesgos y limitaciones que estas nuevas herramientas 2.0 llevan aparejadas. Bernal (2015) cuestiona la pluralidad informativa y habla de efecto bumerang al plantear que los usuarios consumen mayoritariamente contenidos producidos por su círculo de afines. Bruns y Burgess (2012) alertan del posible traslado a las redes de la polarización política que se vive en el entorno tradicional mientras que Hampton et al. (2014) señalan que estas perpetúan la espiral del silencio enunciada por Noelle-Neumann.

\section{Debate social, Twitter y framing}

La evolución de los procesos de producción y distribución informativa ha derivado en la creación de nuevos mecanismos para articular la agenda pública y la forma en la que esta se comunica (Casero-Ripollés y Feenstra, 2012). En este contexto es especialmente relevante el papel desempeñado por Twitter entendido como un sistema de comunicación autónomo en la línea del periodismo ambiental enunciado por Hermida (2010). El contenido generado en esta plataforma se entiende como un todo interpretable bajo los parámetros del "sistema de inteligencia colectiva" (Flores, 2009) que surge a raíz de la conexión entre usuarios. Lo que prima es el contenido compartido más que las características particulares de los usuarios que los producen. Así, Twitter se convierte en un instrumento comunitario (Asur et al., 2011) en el que 
la suma de tuits durante el debate de un tema de actualidad tiene más valor que la información individual (López Meri, 2015).

Autores como De Fleur y Ball-Rockeach (1993) sostienen que el poder político se vincula también con las relaciones de microdependencia que se establecen entre pequeñas redes interpersonales y los medios en el momento en que se procesa la información. Es decir, que también participan del poder los líderes de opinión, ciudadanos y otros grupos sociales que intervienen en el debate público aportando su perspectiva particular sobre un asunto concreto. Con anterioridad, Lazarsfeld et al. (1944) ya habían establecido en el The People's Choice su teoría del two step flow, según la cual los grupos sociales contribuyen a decodificar e interpretar los mensajes de los medios.

A la hora de determinar qué actores forman parte del debate público y cuáles son sus aportaciones resulta especialmente útil el seguimiento de hashtags o etiquetas. Fueron creadas ad hoc por los propios usuarios de Twitter para poder agrupar todos los mensajes relacionados con un mismo evento o tema específico (Carter et al., 2011). Su presencia agrega un mayor contexto a las conversaciones surgidas en la red (Rojo, 2012), otorgan mayor visibilidad a los contenidos allí tratados (Quintana y Tascón, 2015), y ayudan a enmarcar los mensajes al establecer referencias comunes en el discurso (Dahlberg-Grunberg y Lindgren, 2014).

Investigaciones como la de Valera-Ordaz y López (2014) o Palau-Sampio et al. (2017) procesos similares desde la perspectiva del encuadre. Desde hace décadas, el framing se ha convertido en una destacada línea de investigación en el campo de la comunicación. Su punto de partida se encuentra en la noción de marco de referencia sociológico de Goffman (1974), que posteriormente se extendió a otros campos científicos. El concepto de frame como marco organizativo e interpretativo de las noticias se introdujo en el ámbito de la comunicación en los años ochenta para consolidarse con el cambio de siglo (Vicente Mariño y López-Rabadán, 2009). Dentro de los estudios de framing centrados en el mensaje noticioso, destacan los trabajos pioneros de Gans (1980), Tuchman (1983) y Gitlin 1980).

A pesar del creciente volumen de producción científica en este ámbito (ArdévolAbreu, 2015), el framing se resiste a una definición conceptual clara y precisa (Valera-Ordaz, 2016), lo que provoca cierta tensión teórica y metodológica (Sádaba et al. 2012). Para Entman (1993), enmarcar consiste en seleccionar determinados aspectos de la realidad y destacarlos en un texto comunicativo de manera que se promueve una determinada definición de un problema, una interpretación causal, una valoración moral y una recomendación de soluciones. Estas cuatro operaciones discursivas convergen con las tareas diagnósticas (definición, interpretación de la causa y evaluación moral) y prognósticas (propuestas de soluciones) que los estudiosos de los movimientos sociales atribuyen a los marcos de acción colectiva (Benford y Snow, 2000).

\section{Objetivos e hipótesis}

Esta investigación se propone analizar el discurso en Twitter de los principales actores implicados en el cierre de la radiotelevisión pública valenciana (RTVV). Se trata de colectivos que participaron o se vieron involucrados en mayor o menor medida en la decisión de cerrar el ente público valenciano (Tabla 1): 
Tabla 1. Muestra de los actores analizados.

\begin{tabular}{|c|c|c|}
\hline Actor & Cuenta & Cargo \\
\hline \multirow{3}{*}{$\begin{array}{l}\text { Gobierno y } \\
\text { oposición }\end{array}$} & @AlbertoFabra & $\begin{array}{l}\text { Alberto Fabra, presidente de la Generalitat Valen- } \\
\text { ciana }\end{array}$ \\
\hline & @gva_info & $\begin{array}{l}\text { Generalitat Valenciana, órgano de gobierno de la } \\
\text { Comunidad Autónoma }\end{array}$ \\
\hline & $@$ SocialistesVal & $\begin{array}{c}\text { PSPV-PSOE } \\
\end{array}$ \\
\hline \multirow{3}{*}{$\begin{array}{l}\text { Trabajadores } \\
\text { implicados }\end{array}$} & @vicentjuanmtnez & Vicent Juan, periodista RTVV \\
\hline & @amaliasebastian & Amalia Sebastián, periodista RTVV \\
\hline & @vicentmifsud & Vicent Mifsud, periodista RTVV \\
\hline \multirow{3}{*}{$\begin{array}{l}\text { Medios de co- } \\
\text { municación }\end{array}$} & @levante_emv & $\begin{array}{l}\text { Levante El Mercantil Valenciano (Levante-EMV), } \\
\text { periódico líder de la Comunidad Valenciana }\end{array}$ \\
\hline & @informacion_es & $\begin{array}{l}\text { Diario Información o Información, periódico de la } \\
\text { provincia de Alicante }\end{array}$ \\
\hline & @elpais_valencia & $\begin{array}{c}\text { Edición regional del periódico nacional El País } \\
\text { para la Comunidad Valenciana (Valencia, Caste- } \\
\text { llón y Alicante) }\end{array}$ \\
\hline \multirow{3}{*}{$\begin{array}{l}\text { Universidades } \\
\text { públicas }\end{array}$} & @UJI_noticias & Universitat Jaume I de Castellón \\
\hline & @UV_EG & Universitat València \\
\hline & @UA_Universidad & Universidad de Alicante \\
\hline
\end{tabular}

Fuente: Elaboración propia.

- Gobierno y oposición: se les ha elegido como protagonistas políticos del cierre del ente público valenciano. El gobierno a través de su presidente tomó la decisión de cerrar y la llevó a cabo a pesar de las protestas. Interesa saber qué argumentó al respecto y comparar el discurso con el del principal partido de la oposición en aquel momento, los socialistas valencianos, como contrapunto.

- Trabajadores de RTVV: se les ha elegido por ser el colectivo más directamente afectado por el cierre del ente público valenciano, al quedarse sin trabajo. Se analizan los perfiles de tres de los periodistas más influyentes de acuerdo con la herramienta de monitorización Social Mention ${ }^{4}$.

- Medios de comunicación: se les ha elegido como actores cuyo discurso permite a la sociedad construir la realidad (Grossi, 1985). También, para comprobar cómo reaccionan ante el cierre de otro medio de comunicación que, además, es público y por lo tanto, tiene asociados unos valores determinados. Se han escogido los tres diarios que informan sobre la Comunidad Valenciana con mayor difusión en ella durante el 2013 de acuerdo con el Estudio General de Medios (EGM, 2013).

\footnotetext{
4 Social Mention es una herramienta que permite conocer qué se comenta en relación a un tema, marca o persona en Internet. En este caso, se buscaron los perfiles más influyentes en la conversación sobre RTVV en Twitter.
} 
- Universidades públicas de la Comunidad Valenciana en las que se ofertan estudios de Comunicación: se les ha elegido por el impacto que el cierre de RTVV puede provocar en el futuro de estos centros de enseñanza y, por ende, en los profesionales de la comunicación que allí se forman.

- Para garantizar que la muestra sea lo más homogénea posible, se han seleccionado tres actores de cada una de las dimensiones antes mencionadas.

Este artículo se propone los siguientes objetivos:

a) Determinar los temas de los hablan en Twitter los principales protagonistas del cierre de RTVV.

b) Analizar las funciones de encuadre adoptadas por cada uno de ellos durante el proceso del cierre del ente público valenciano.

c) Analizar el empleo de los hashtags contenidos en los tuits.

Nuestra hipótesis de partida es que el debate en Twitter sobre el cierre de RTVV se centra en los mismos temas con independencia del colectivo, pero cada protagonista lo enmarca de manera diferente. Las cuestiones vinculadas con la atribución de responsabilidades por lo sucedido, la pérdida de un servicio público de promoción de la cultura autóctona así como las demandas de una radiotelevisión pública de calidad prevalecen en el discurso de los periodistas afectados mientras que el resto de actores se centra preferentemente en explicar qué ha ocurrido.

\section{Metodología}

Esta investigación aplica la técnica metodológica del análisis de contenido. La muestra está compuesta por las cuentas oficiales en Twitter de los principales implicados en el proceso, anteriormente justificados y recogidos en la ya referida Tabla 1.

Se ha escogido Twitter al entenderse como una de las principales herramientas de trabajo para los profesionales a la hora de contactar con potenciales fuentes y buscar información (Carrera et al., 2012). Además, en el último informe disponible hasta la fecha del Instituto Reuters (2016) se señala la importancia de las redes sociales en general y de Twitter en particular como fuente de información para los internautas.

El trabajo de campo comprende los cuatro días claves del proceso, que se corresponden al momento en que se anunció el cierre de la cadena (6 y 7 de noviembre) y su clausura efectiva ( 29 y 30 de noviembre). Se ha pretendido capturar y analizar los mensajes que fueron difundidos en los momentos de "mayor trauma colectivo" (Berná, Pérez y Arroyas, 2013), es decir, más cercanos al hecho clave o motor que suscita el debate en cuestión. Durante este tiempo, se recopilaron un total de 1.014 mensajes.

El proceso de captura se realizó de manera manual una semana después de su difusión con el objetivo de poder captar y analizar todo su recorrido. La unidad de análisis fue un tuit. Se ha optado por seleccionar solo aquellos que aludían específicamente al cierre de RTVV, ya sea en el cuerpo del mensaje, en los hashtags o en los recursos empleados (como fotografías, vídeos o cualquier otro tipo de contenido multimedia). 
A continuación, se procede a desgranar bajo qué criterios, objetivos y codificaciones se ha aplicado la metodología de análisis seleccionada a cada unidad de análisis. Resulta conveniente resaltar que, en todos los casos, han sido ambas investigadoras las únicas participantes en la codificación, realizando el proceso de manera conjunta.

Tabla 2. Protocolo de análisis de contenidos.

\begin{tabular}{|c|c|}
\hline Tipo & Contenido \\
\hline Protesta & $\begin{array}{c}\text { Tuits que manifiestan rechazo o desazón por perder } \\
\text { un servicio público de radiotelevisión }\end{array}$ \\
\hline Información & $\begin{array}{c}\text { Tuits que se limiten a exponer de manera aséptica lo que está } \\
\text { sucediendo con respecto al cierre de RTVV }\end{array}$ \\
\hline Movilización & $\begin{array}{c}\text { Tuits que llaman a la manifestación o facilitan datos sobre dónde y } \\
\text { cuándo manifestarse }\end{array}$ \\
\hline Apoyo & $\begin{array}{c}\text { Tuits que muestran solidaridad con los trabajadores } \\
\text { afectados por el cirre }\end{array}$ \\
\hline
\end{tabular}

Fuente: Elaboración propia.

Tabla 3. Protocolo de análisis de contenidos y temas.

\begin{tabular}{|c|c|c|}
\hline Variable & \multicolumn{2}{|c|}{ Tema } \\
\hline \multirow{6}{*}{ Protesta } & \multicolumn{2}{|c|}{$\begin{array}{l}\text { Crítica política, en la que se cuestiona la decisión de Fabra o se manifesta } \\
\text { disconformindad con su decisión }\end{array}$} \\
\hline & \multicolumn{2}{|c|}{ Demanda de una RTVV de calidad } \\
\hline & \multicolumn{2}{|c|}{ Demanda de justicia y petición de responsabilidades } \\
\hline & \multicolumn{2}{|c|}{ Quejas por el trato dispensado a los trabajadores } \\
\hline & \multicolumn{2}{|c|}{ Mensajes que reflejan actos de protesta } \\
\hline & \multicolumn{2}{|c|}{$\begin{array}{l}\text { Mensajes que señalan la pérdida de un instrumento de promoción y } \\
\text { difusión cultural (en este caso, de la lengua y cultura valenciana) }\end{array}$} \\
\hline \multirow{9}{*}{ Información } & \multicolumn{2}{|c|}{ Mensajes que enlazan a streaming o últimas horas } \\
\hline & \multirow{2}{*}{$\begin{array}{l}\text { Mensajes que explican posturas sobre } \\
\text { la toma de decisiones sobre el cierre }\end{array}$} & Justificación de la decisión \\
\hline & & Reacciones a la decisión \\
\hline & \multicolumn{2}{|c|}{ Mensajes que aportan datos de contexto sobre el cierre del ente público } \\
\hline & \multirow{4}{*}{ Mensajes con declaraciones textuales } & Ataques a Fabra y a su decisión \\
\hline & & $\begin{array}{l}\text { Apelación a la identidad como } \\
\text { pueblo }\end{array}$ \\
\hline & & $\begin{array}{l}\text { Promesa electoral de } \\
\text { recuperación }\end{array}$ \\
\hline & & Compromiso hacia RTVV \\
\hline & \multicolumn{2}{|c|}{ Mensajes que informan sobre lo que ocurre en tiempo real } \\
\hline \multirow{2}{*}{ Movilización } & \multicolumn{2}{|c|}{ Mensajes que llaman a la movilización } \\
\hline & \multicolumn{2}{|l|}{ Mensajes que llaman a la manifestación } \\
\hline \multirow{4}{*}{ Apoyo } & \multicolumn{2}{|c|}{ Mensajes que manifiestan solidaridad con los trabajadores } \\
\hline & \multicolumn{2}{|c|}{ Mensajes que agradecen las muestras de solidaridad } \\
\hline & \multicolumn{2}{|c|}{ Mensajes que incluyen fotografía o vídeo a modo de testimonio } \\
\hline & \multicolumn{2}{|c|}{ Mensajes que agradecen el trabajo de los periodistas } \\
\hline
\end{tabular}

Fuente: Elaboración propia. 


\subsection{Análisis de los contenidos y temas}

La investigación busca determinar el tipo de contenido publicado por los actores analizados a través de su cuenta en Twitter y la temática que predomina en cada uno de ellos. Por eso, se han diseñado dos fichas de análisis complementarias (Tabla 2 y Tabla 3) a partir de los objetivos de la investigación y de la literatura académica publicada hasta el momento sobre la materia, entre las que ocupan un lugar destacado Van Laer y Van Aelst (2010) y Bennet y Segerberg (2012). Las cuatro tipologías recogidas se han deducido de los trabajos de Alzamora y Braga (2014), Cárcar Benito (2015) y Scherman, Arriaga y Valenzuela (2013) para la categoría de protesta; de Vallespín (2011) y Castells (2012) para la de movilización; y de Bennett y Segerberg (2014) para la de apoyo.

\subsection{Análisis de los enfoques discursivos de los mensajes y de los hashtag}

La ficha de análisis aplicada se ha basado en las cuatro funciones básicas de encuadre enunciadas por Entman (1993): definición del problema, atribución de responsabilidad, valoración moral y recomendación de tratamiento. Se ha optado por clasificar cada uno de los tuits sólo en una de las cuatro funciones posibles de acuerdo con su contenido principal. Esta misma plantilla de análisis (Tabla 4) también se ha utilizado para determinar el uso de los hashtag.

En el caso de los hashtags es preciso señalar que el análisis se ha limitado a aquellas etiquetas que contenían la suficiente información como para atribuirles funciones de encuadre. No son susceptibles de análisis, por ejemplo, etiquetas como \#Canal9 o \#RTVV.

Tabla 4. Función y descripciones.

\begin{tabular}{|c|c|}
\hline Función & Descripción \\
\hline $\begin{array}{l}\text { Definición del } \\
\text { problema }\end{array}$ & $\begin{array}{l}\text { Tuits que explican y contextualizan el cierre de RTVV } \\
\text { (sin valoración) }\end{array}$ \\
\hline $\begin{array}{l}\text { Atribución de } \\
\text { responsabilidad }\end{array}$ & $\begin{array}{l}\text { Tuits que identifican posibles responsables del hecho en sí } \\
\text { (el cierre del ente público) }\end{array}$ \\
\hline Valoración moral & $\begin{array}{c}\text { Tuits que justifican el cierre de la corporación o que lo relacionan } \\
\text { con el sentimiento de pérdida de la cultura y los valores } \\
\text { valencianos }\end{array}$ \\
\hline $\begin{array}{l}\text { Recomendación de } \\
\text { tratamiento }\end{array}$ & $\begin{array}{c}\text { Tuits que aportan posibles soluciones como la reapertura de } \\
\text { RTVV o informan de lo que se está haciendo para conseguirlo } \\
\text { (manifestaciones, protestas...) }\end{array}$ \\
\hline
\end{tabular}

Fuente: Elaboración propia de acuerdo con Entman (1993).

\section{Resultados}

\subsection{Temas de los mensajes por tipos de contenidos}

Tras analizar los 1.014 tuits que conforman la muestra, se observa que casi la mitad de los mensajes se corresponde a contenidos de tipo informativo $(43,39 \%)$, seguidos 
por los de protesta $(29,48 \%)$, apoyo $(17,35 \%)$ y movilización $(9,76 \%)$. En cuanto a los temas, destaca muy por encima del resto los englobados dentro de la categoría “¿Qué está pasando?” (27,4\% del total de tuits), que se ocupa de explicar la última hora de lo que acontecía. A continuación, están los mensajes que se lamentan del cierre del ente público por la supuesta pérdida de un instrumento de promoción y difusión cultural del valenciano $(11,8 \%)$, seguido a escasa distancia por los que critican políticamente lo ocurrido $(11,7 \%)$. Después, los tuits que manifiestan solidaridad con los trabajadores afectados $(6,5 \%) \mathrm{y}$, por último, los que recogen declaraciones textuales con ataques a la decisión $(6,1 \%)$. Pasamos ahora a desglosar los temas por tipos de contenidos.

\subsubsection{Temas vinculados con la información}

Desde el punto de vista informativo (Gráfico 1), Twitter es empleado mayoritariamente $(63,2 \%)$ para trasladar la última hora en relación al cierre, movilizaciones y reacciones en torno al final de RTVV. A esto se añaden los mensajes que ofrecen la posibilidad de acceder a la retransmisión en directo o vía streaming a lo que está pasando $(3,41 \%)$. Un ejemplo de ello son los tuits que incluyen enlaces a comparecencias, manifestaciones o el último día de emisiones de la corporación. Los autores que más contribuyen a relatar en directo qué es lo que está ocurriendo y en cierto modo fijar así las coordenadas que delimitan el problema son los medios de comunicación y los periodistas afectados por el cierre $(24,7$ y $24,3 \%$, respectivamente).

Gráfico 1. Temas de los tuits de tipo informativo.

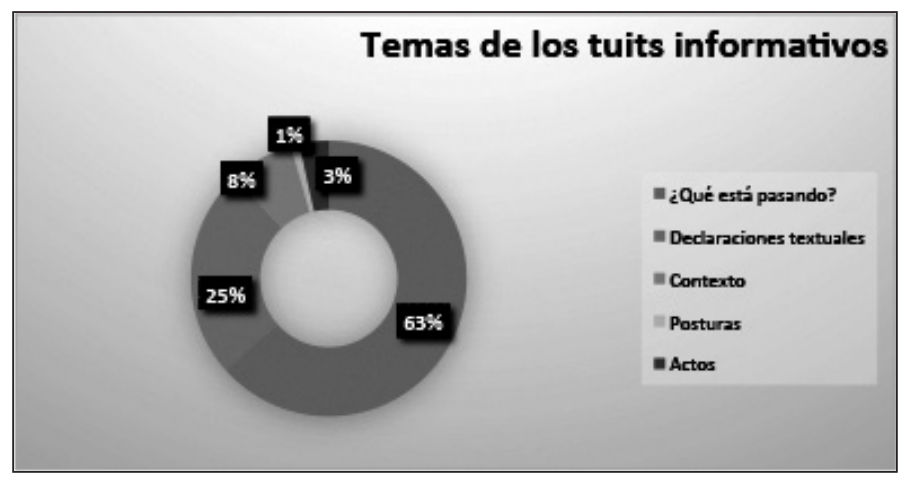

Fuente: Elaboración propia.

El segundo uso prioritario de Twitter en este mismo apartado es reproducir declaraciones textuales de los actores implicados en el cierre. Aquí, son las citas que incluyen una crítica hacia la gestión de Fabra y la Generalitat las que tienen mayor presencia. El 62,62\% de las publicaciones correspondientes a este tema se adscriben a esta variable. De ellas, el 52,5\% han sido difundidas a través de la cuenta oficial en Twitter del PSPV-PSOE, el $9 \%$ por cuentas de los periodistas y un $1 \%$ por el diario Levante. También se han identificado 14 mensajes que recogen declaraciones textuales que intentan justificar el cierre de RTVV. Todos menos uno proceden de cuentas 
vinculadas a los medios de comunicación analizados, que en este debate en Twitter se centran más en difundir la postura del Gobierno de la Generalitat y de su Presidente, que del resto de protagonistas. En general, los tuits que apelan a la identidad y al compromiso por una televisión pública de calidad son minoritarios: el 11,11 y el $4,04 \%$, respectivamente.

El tercer tema más tratado dentro de esta categoría tiene que ver con aportar información de contexto sobre la Corporación, como por ejemplo datos sobre sus audiencias, su evolución, artículos de opinión o análisis de especialistas. En este caso, son las cuentas de los periodistas $(80,64 \%)$ y medios de comunicación $(13,90 \%)$ las que explotan de manera más notable este uso.

En último lugar, encontramos aquellos posts en los que los actores implicados manifiestan en primera persona su postura. Aquí destacan los tres únicos tuits publicados por el Presidente de la Generalitat, Alberto Fabra (Imagen 1), en los que se explica el porqué de su decisión y que fueron ampliamente retuiteados.

Imagen 1. Postura oficial del presidente de

la Generalitat en relación al cierre de RTVV.

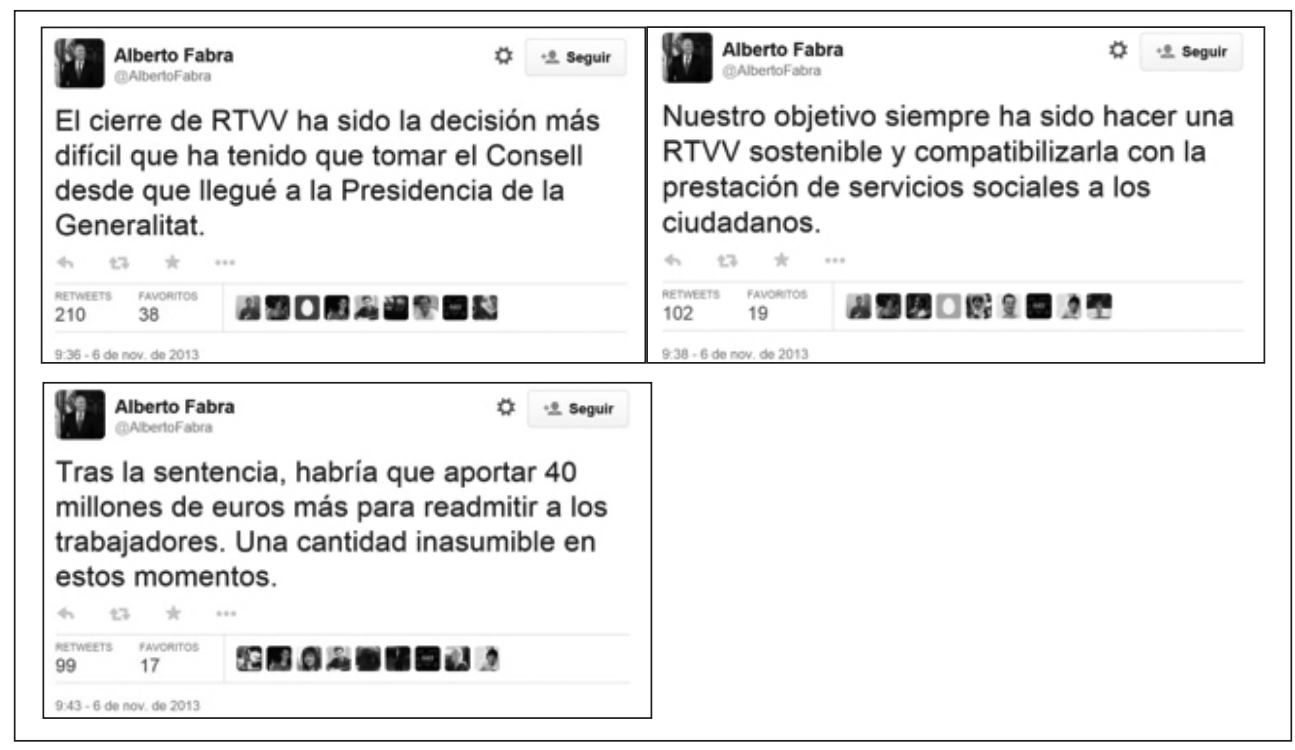

\subsubsection{Temas vinculados con la protesta}

Desde el punto de vista de la protesta, los mensajes se ocupan fundamentalmente de dos cuestiones que suponen el $80 \%$ de la muestra total de este apartado (Gráfico 2): evocar la importancia del ente público en la vertebración del espacio social y cultural valenciano $(40,13 \%)$ y criticar la decisión tomada por la Generalitat con respecto al cierre de RTVV $(39,8 \%)$. Los resultados alcanzados por el resto de variables consideradas sólo ascienden, por lo tanto, al $20 \%$ del total de mensajes. 
Gráfico 2. Temas de los tuits de tipo protesta.

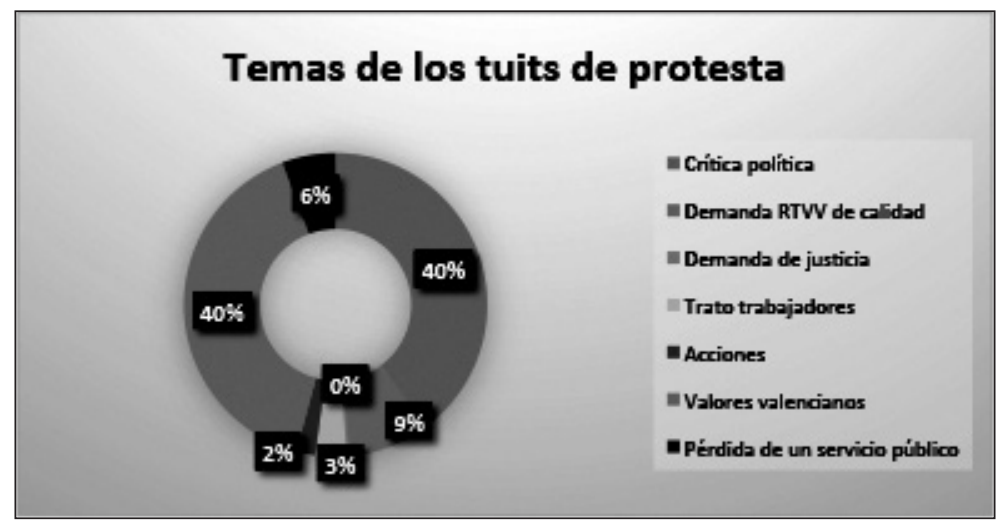

Fuente: Elaboración propia.

Los actores dominantes del discurso de protesta son los periodistas, autores del $89,2 \%$ del total de tuits de esta categoría, seguidos a mucha distancia, por los políti$\cos (9,6 \%)$. Los argumentos del primer colectivo se centran en la pérdida de la radiotelevisión pública valenciana como referente de información de proximidad. Así lo demuestran las publicaciones que aluden a que, a partir del cierre, no habrá ningún medio que informe sobre cuestiones propias de la comunidad como las Fallas, las Fogueres, las muixerangues o los Pelegrins de les Useres. Ni tampoco, que ayude a construir un espacio cultural común valenciano. Destacan aquí los tuits que evocan programas clásicos de la parrilla de la radiotelevisión en lengua valenciana como "A la babalà", “ "La Taula esportiva” o "Encontres”.

\subsubsection{Temas vinculados con el apoyo}

Dentro de los tuits catalogados como apoyo (Gráfico 3), destacan aquellos que se centran en mostrar explícitamente solidaridad con los trabajadores afectados por el cierre del ente público. El 37,5\% de las publicaciones ubicadas aquí encajan con este patrón. Mientras, aquellas en las que los periodistas agradecen estas muestras de cariño ascienden al 26,13\%. A muy poca distancia, encontramos los tuits que incluyen contenido audiovisual $(21,59 \%)$ que ayuda a reforzar este mensaje a modo de fotografía o vídeo testimonial. Por último, se sitúan aquellos que buscan reconocer el trabajo desempeñado por los profesionales del medio clausurado $(14,77 \%)$.

En este tipo de contenido, los actores que muestran mayor implicación son los periodistas, especialmente activos a la hora de mostrar solidaridad con sus propios compañeros (ya sea a través de tuits propios o retuiteando los de terceras personas) y agradecer las muestras de apoyo recibidas. El resto de protagonistas del debate en Twitter optan por el silencio, al no corresponder ninguno de sus mensajes a esta categoría. Las únicas excepciones son un tuit de apoyo a los trabajadores retuiteado por Información, y siete de las 172 publicaciones del PSPV-PSOE en las que sí se explicita el apoyo a este colectivo. 
Gráfico 3. Temas de los tuits de tipo apoyo.

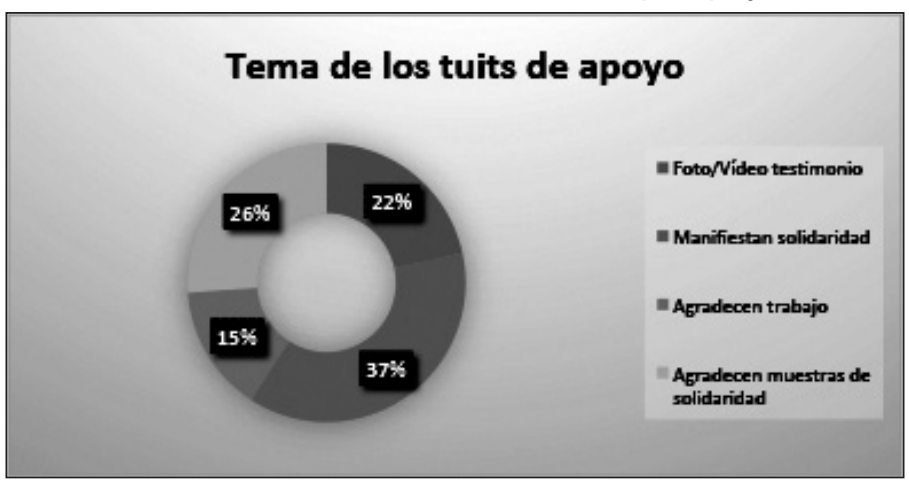

Fuente: Elaboración propia.

\subsubsection{Temas vinculados a la movilización}

La última categoría en cuanto a volumen de mensajes se corresponde con la tipología de movilización. Esta capacidad es una de las potencialidades que, habitualmente, se destaca de las redes sociales (Castells, 2012). Sin embargo, en el caso del cierre de RTVV, esta función no es la prioritaria entre los actores implicados (GonzálezMolina y Ramos del Cano, 2015). De hecho, el volumen de tuits que se corresponde a esta categoría es el menor de todos los analizados $(9,76 \%)$.

La mayoría de mensajes publicados aquí son llamamientos a los ciudadanos para que se movilicen en contra del cierre de RTVV, a favor de un servicio público de radiotelevisión de calidad o reclamando acciones concretas para sancionar a los que hacen responsables del final del ente público (Gráfico 4). El llamamiento a manifestaciones en contra del cierre de RTVV es el segundo gran tema en el que se dividen los contenidos de la categoría de movilización, con mensajes en los que se pide a los ciudadanos que se concentren aportándoles, además, la hora y el lugar del evento.

Gráfico 4. Temas de los tuits de tipo movilización.

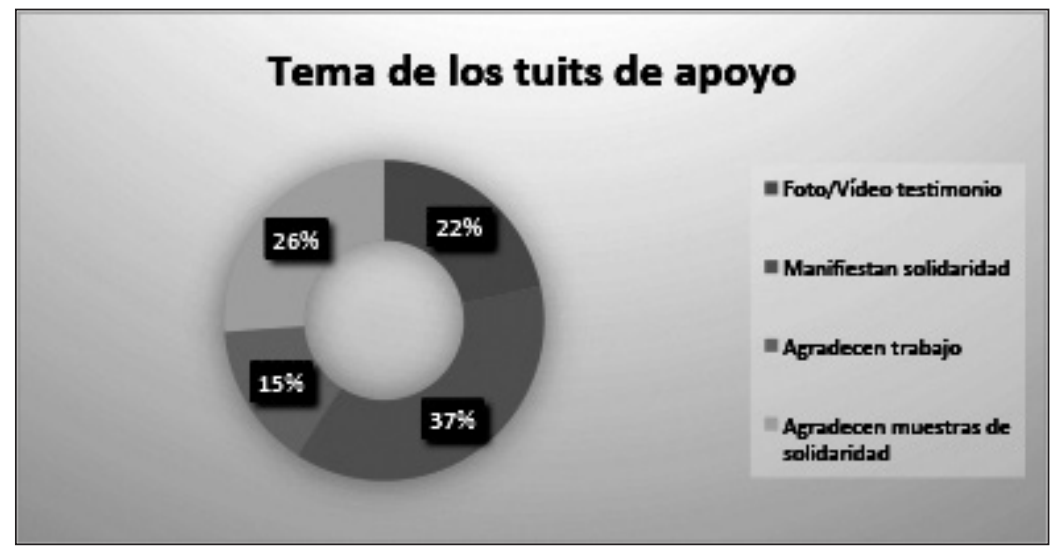

Fuente: Elaboración propia. 
El colectivo más activo en ambos casos es el de los periodistas implicados en el cierre del ente público, que firman el $92,92 \%$ de los tuits encuadrados dentro de esta variable. El resto se reparten entre el PSPV-PSOE $(4,04 \%)$ y Levante $(3,03 \%)$.

\subsection{Encuadres de los protagonistas del cierre de RTVV}

Una vez detectados los tipos de contenidos y temas que predominan en el debate en Twitter sobre el cierre de RTVV, pasamos a centrarnos en la manera en que cada colectivo protagonista enmarca esta cuestión a partir de las funciones de encuadre de Entman (1993) anteriormente enumeradas.

En general, el debate se centra más en valorar moralmente lo que sucede que en buscar causas y aportar soluciones (Tabla 5). Destacan, en este sentido, los mensajes que muestran solidaridad con los trabajadores que pierden su trabajo con el cierre de RTVV, que califican la situación en un sentido u otro o que apelan a la necesidad de que vuelva un servicio público de radiotelevisión alegando motivos culturales y lingüísticos. Las cuentas de los periodistas Vicent Juan y la de los socialistas valencianos (PSPV-PSOE) son las que más mensajes acumulan en esta categoría con un $41,6 \%$ y un $18,4 \%$, respectivamente.

Tabla 5. Identificación de las funciones básicas

de encuadre en porcentajes sobre el total $(\mathrm{N}=1.014)$.

\begin{tabular}{|l|c|}
\hline Funciones de encuadre & Total \\
\hline Definición del problema & $33,8 \%$ \\
\hline Diagnóstico de causas & $\mathbf{7 \%}$ \\
\hline Valoración moral & $44,2 \%$ \\
\hline Propuesta de soluciones & $14,8 \%$ \\
\hline Total & $\mathbf{1 0 0 \%}$ \\
\hline
\end{tabular}

Fuente: Elaboración propia.

El debate en Twitter de los protagonistas del cierre de RTVV se nutre también de mensajes cuya única finalidad es ofrecer información en tiempo real sobre lo que ocurría en esos días, como los directos. Nuevamente, la cuenta del periodista Vicent Juan sobresale en este sentido (31,7\%) junto a la del periódico Levante $(22,7 \%)$. Las propuestas de soluciones que se plantean pasan por animar a protestar públicamente para conseguir reabrir el ente público o solicitar directamente su reapertura. Es especialmente así entre los periodistas como Vicent Juan (48,3\%) o Vicent Midsuf $(18,5 \%)$. En cuanto a la búsqueda de responsables, con menor volumen de tuits, los mensajes se centran básicamente en culpar al presidente de la Generalitat, Alberto Fabra, y al PP de lo sucedido, a los que atribuyen un cierto desprecio por lo público y la hipotética destrucción del autogobierno valenciano.

Las cuentas corporativas de la Generalitat Valenciana y la del presidente de la Comunitat apenas se refieren al cierre de RTVV en los días analizados. De hecho, todos los mensajes detectados sobre este tema son del primer día. Para ambos actores, se trata de una decisión inevitable debida a causas económicas (la imposibilidad de readmitir a los trabajadores tras un ERE declarado nulo). Los mensajes aluden a la voluntad de priorizar las políticas sociales, a la dificultad de tomar la decisión de 
cierre y a que siempre se ha buscado una radiotelevisión pública sostenible compatible con la prestación de servicios sociales a los ciudadanos. En este contexto, la única solución es el cierre.

Por el contrario, el enmarcado del PSPV-PSOE se centra en destacar la supuesta falta de independencia política del gobierno valenciano respecto del central. Para los socialistas valencianos, el cierre de la corporación es una decisión tomada desde Madrid y, al contrario de lo que argumenta Fabra, sí que consideran viable la pervivencia del ente público con las políticas sociales. Se trataría meramente de un problema político del cual Fabra y el PP en su conjunto serían los únicos responsables. Eximen, pues de culpa los trabajadores a los que muestran su apoyo. Los mensajes publicados vinculan el cierre de RTVV con la corrupción y con el desmantelamiento del autogobierno valenciano, ya que entienden que la radiotelevisión pública es un instrumento de vertebración de la cultura autóctona. Por eso, califican el cierre de RTVV como golpe de estado mediático. Como soluciones, proponen disolver las Cortes para convocar nuevas elecciones, exigir a la Fiscalía que se depuren responsabilidades políticas y jurídicas, la apertura de una comisión de investigación y reabrir la televisión una vez ellos alcancen el poder para lo que instan a no votar al PP. Durante estos días, además, presentaron una suspensión cautelar ante el TSJCV por los daños irreparables que a su juicio iba a provocar el cierre de RTVV.

Los medios de comunicación analizados básicamente recogen las acciones y argumentos de los principales protagonistas del cierre de RTVV a través de la narración de lo que aconteció durante los días clave del proceso. Mientras que los demás actores inician su relato hablando de las consecuencias o posicionándose a favor o en contra, los medios lo empiezan con el cierre de Canal 9. Después, desgranan lo que va ocurriendo incluyendo los puntos de vista de políticos, trabajadores, gente de la calle y personalidades del mundo cultural. Los tres medios analizados coinciden en describir los días posteriores al cierre como caóticos hablando incluso de una rebelión por parte de los trabajadores lo que, para medios como El País, equivale a una "primavera informativa". Tanto la atribución de causas como la valoración moral o la propuesta de soluciones al cierre, se realiza de manera indirecta ya que los medios recogen las opiniones, propuestas, justificaciones y argumentos del resto de actores implicados. Los tres medios aluden a una posible censura existente en RTVV y son los que más abiertamente hablan del conflicto entre periodistas y gobierno valenciano en relación al cierre del ente público.

Los mensajes de los periodistas afectados por el cierre de RTVV se centran en tres aspectos: agradecer las muestras de solidaridad y apoyo que reciben por el cierre de la corporación, lamentar la pérdida de un espacio comunicativo valenciano desde el que informar de acontecimientos de proximidad como las fogueres, la Magdalena o las Fallas, y vincular al ente público valenciano con la vertebración de un espacio comunicativo propio. En sus tuits, los periodistas dejan claro su orgullo por trabajar en el ente público e insisten en que no son los culpables del cierre, responsabilidad que atribuyen al presidente de la Generalitat. Entienden que el final de RTVV ha provocado una catarsis en la sociedad valenciana y relacionan los hechos que están viviendo con lo sucedido la noche del $23-\mathrm{F}$. Como soluciones, proponen firmar una iniciativa en la plataforma change.org para evitar el cierre de RTVV, organizar una 
huelga general en protesta, convocar nuevas elecciones y movilizar a los ciudadanos para que se manifiesten en contra del cierre de la corporación.

Las universidades públicas valencianas con estudios en Comunicación apenas mencionan el cierre de RTVV en sus cuentas corporativas de Twitter. Su discurso es el más pobre de todos los analizados. De hecho, una de ellas (Universidad de Alicante) ni siquiera hace mención a ello. No hay ningún mensaje que informe expresamente del final del ente público valenciano sino que se hace de manera indirecta posicionándose en contra (valoración moral) o pidiendo una radiotelevisión pública de calidad (posible solución). No hay alusiones a las posibles causas o responsables, ni al colectivo de periodistas afectados ni a otras cuestiones vinculadas con la promoción de la cultura y lengua valenciana. Tampoco hay propuestas de solución para evitar la desaparición del ente público más allá de la ya comentada de pedir un medio público de calidad.

\subsection{El debate a través de los hashtags}

Analizar los hashtags empleados por los actores implicados en el debate sobre la clausura de RTVV era el último de nuestros objetivos en este trabajo. El 89,1\% de los 1.014 mensajes publicados en Twitter durante los cuatro días clave de este proceso contienen una etiqueta, cosa que denotaría un uso avanzado de la plataforma por parte de los protagonistas del cierre. Con todo, existen notables diferencias entre unos y otros (Tabla 6). El colectivo de los periodistas se desmarca de nuevo respecto del resto al ser el que en más ocasiones usa estas etiquetas $(68,9 \%)$ al contrario de las universidades, que no utilizan ninguna. Además, los periodistas también son los actores que mayor variedad de hashtags utilizan en sus mensajes, combinando diversas etiquetas en sus tuits.

Tabla 6. Uso de hashtags por parte de los protagonistas del debate.

\begin{tabular}{|l|c|c|c|c|c|}
\hline & Periodistas & Universidades & Medios & Políticos & Total \\
\hline $\begin{array}{l}\text { Hashtags } \\
\text { totales }\end{array}$ & 623 & 0 & 88 & 193 & 904 \\
\hline $\begin{array}{l}\text { Variedad de } \\
\text { hashtags }\end{array}$ & 74 diferentes & 0 diferentes & 7 diferentes & 20 diferentes & $\begin{array}{c}89 \\
\text { diferentes }\end{array}$ \\
\hline
\end{tabular}

Fuente: Elaboración propia.

Los 12 actores analizados emplean un total de 89 hashtags diferentes. Los 30 con mayor peso se recogen en la Tabla 7. De este total, los periodistas emplean 74 mientras que los políticos usan una veintena y los medios, 7. Los cinco hashtag más utilizados por los trabajadores afectados por el cierre son \#RTVVnoestanca (344 ocasiones), \#SíaRTVV (75), \#RTVV (44), \#RTVVtornara (31) y \#BCNambRTVV y \#Faltalataula (8 veces en los dos casos). Los políticos son el segundo colectivo que más variedad de etiquetas utiliza en sus mensajes. La más abundante es, nuevamente, \#RTVVnoestanca (87 ocasiones), seguida de \#RTVVexecutada y \#FabraEREsincapaz (25 veces cada una), \#RTVV (16) y \#FabraDimissio (13). Los medios, por su parte, acompañan sus mensajes de manera más habitual con las etiquetas \#RTVV (77 veces), \#Canal9 (4), \#RTVVnoestanca (2) y \#Valencia (1). 
Tabla 7. Uso de hashtags por parte de los protagonistas del debate ${ }^{5}$.

\begin{tabular}{|l|c|c|c|c|c|}
\hline \multicolumn{1}{|c|}{$\#$} & Universidades & Políticos & Periodistas & Medios & Total \\
\hline RTVVnoestanca & 0 & 87 & 344 & 2 & 433 \\
\hline RTVV & 0 & 16 & 44 & 77 & 137 \\
\hline SiaRTVV & 0 & 0 & 75 & 0 & 75 \\
\hline RTVVtornara & 0 & 5 & 31 & 0 & 36 \\
\hline RTVVexecutada & 0 & 25 & 7 & 0 & 32 \\
\hline FabraEREsincapaz & 0 & 25 & 0 & 0 & 25 \\
\hline FabraDimissio & 0 & 13 & 6 & 0 & 19 \\
\hline BCNambRTVV & 0 & 0 & 8 & 0 & 8 \\
\hline Faltalataula & 0 & 0 & 8 & 0 & 8 \\
\hline Canal9 & 0 & 1 & 3 & 4 & 8 \\
\hline Nou & 0 & 0 & 6 & 0 & 6 \\
\hline FabraERESIncapaç & 0 & 6 & 0 & 0 & 6 \\
\hline LladrEROs & 0 & 1 & 4 & 0 & 5 \\
\hline BonDiaCV & 0 & 0 & 4 & 0 & 4 \\
\hline JusticiaRTVV & 0 & 1 & 3 & 0 & 4 \\
\hline BondiaRTVV & 0 & 0 & 4 & 0 & 4 \\
\hline Falles & 0 & 0 & 4 & 0 & 4 \\
\hline rtvvNECESSÀRIA & 0 & 0 & 4 & 0 & 4 \\
\hline NoaltancamentdeRTVV & 0 & 0 & 3 & 0 & 3 \\
\hline TV3 & 0 & 0 & 3 & 0 & 3 \\
\hline Oresponsables & 0 & 3 & 0 & 0 & 3 \\
\hline Valencia & 0 & 0 & 1 & 1 & 2 \\
\hline BonMatiNOUradio & 0 & 0 & 2 & 0 & 2 \\
\hline Benissa & 0 & 0 & 2 & 0 & 2 \\
\hline ocuppyRTVV & 0 & 0 & 2 & 0 & 2 \\
\hline Sinperiodismonohaydemo- \\
cracia & 0 & 0 & 2 & 0 & 2 \\
\hline NoaltancamentdeCanal9 & 0 & 0 & 2 & 0 & 2 \\
\hline CanalNou & 0 & 1 & 1 & 0 & 2 \\
\hline NouRadio & 0 & 193 & 623 & 88 & 904 \\
\hline Ppotiflers & 0 & & 0 & 2 \\
\hline Otros & 0 & 0 & 0 & 4 \\
\hline & 0 & 0 & 2 & 0 & 0 \\
\hline
\end{tabular}

Fuente: Elaboración propia.

\footnotetext{
5 En el apartado Otros se incluyen los hastags que aparecen sólo en una ocasión. Se trata de: \#FabraIncapaç, \#vergonya, \#PP, \#NoCallem, \#EixiremalCarrer, \#lladres, \#NoVotesPP, \#Morella, \#FabraDemagogo, \#Grecia, \#ERT, \#Rosa, \#CAM, \#Humor, \#Ortifus, \#CompolUJI, \#LosJuevesASol, \#30N, \#Valenta, \#VosVull, \#ideas, \#EnstallenRTVV, \#Aixóesprecís, \#MarcaEspana, \#ihaveadream, \#periodisme, \#Noaltancament, \#Mascletà, \#Consell, \#Generalitat, \#Periodismo, \#AR, \#Comunidades, \#Cultura, \#Ayuda, \#Sanidad, \#Economía, \#Madrid, \#RTVVnosecierra, \#SintonizaNou, \#amuntRTVV, \#Magdalena, \#estemvius, \#volemRTVV, \#LaNostraMemoria, \#HistoriaValenciana, \#Corts, \#Gandia, \#SíalValencià, \#UJI, \#recuerdaRTVV, \#Información, \#broma, \#vergoyadepresident, \#vergonyadeFabra, \#Telemadrid Justicia\#, \#FueraPP, \#EleccionsanticipadesJ.
} 
Al examinar los hashtags desde el punto de vista de las funciones de encuadre, existe un volumen destacable de etiquetas como \#Canal9 o \#RTVV que no han sido consideradas en el análisis total al no tener entidad suficiente. Sin embargo, ayudan a contextualizar el tuit al indicar el tema genérico del cual se habla sin aportar carga valorativa.

En general, las etiquetas usadas en los tuits analizados identifican al presidente de la Generalitat, Alberto Fabra, como único responsable del cierre del ente público valenciano aludiendo a su incapacidad en el cargo para gestionar RTVV. Desde el punto de vista de la valoración moral, destacan ejemplos como \#FabraDemagogo \#vergonyadepresident o \#vergonyadeFabra. Con todo, también hay etiquetas que buscan enviar un mensaje de ánimo y solidaridad como \#AmuntRTVV, \#BCNambRTVV o \#Vos Vull. Las propuestas de soluciones pasan por pedir la dimisión de los considerados responsables del cierre del ente (\#Fabradimissio), cambios a nivel político (\#novotesPP o \#eleccionsanticipadesja), el mantenimiento de RTVV (\#RTVVnoestanca o \#SiaRTVV) o la movilización (\#Eixiremalcarrer o \#ocuppyRTVV).

Si se examina el uso de los hashtags por actores, los políticos tienden a incluir etiquetas que valoran moralmente el cierre de RTVV y atribuyen responsabilidades mientras que los periodistas valoran y recomiendan un posible tratamiento o solución. Por su parte, los medios prefieren usar las etiquetas para contextualizar de manera aséptica sus informaciones. Por último, las universidades no emplean hashtags para enriquecer sus mensajes.

\section{Conclusiones}

El análisis de los tuits publicados sobre el cierre de RTVV en Twitter permite concluir que el debate generado por los cuatro protagonistas se centró en informar sobre lo que ocurría, confirmándose así parte de la hipótesis inicial. El uso de los hashtags parece seguir la misma lógica, ya que también apuesta por la información, por lo que el recurso de la etiqueta serviría para reforzar el tema principal del que hablan los tuits.

Los temas que más espacio ocupan son los relacionados con explicar el proceso de cierre y sus vicisitudes en tiempo real, lamentar la pérdida de un espacio comunicativo en lengua valenciana y la crítica política por el cierre del ente público. Pero, al examinar los contenidos por actores, la manera de enfocar este debate cambia. Así, la Generalitat Valenciana califica el cierre de inevitable por tratarse de una cuestión económica en la que las políticas sociales tienen prioridad. Por el contrario, la oposición considera compatible la existencia de un ente público de calidad con el desarrollo de políticas sociales eficaces. Para ellos, el gobierno de Fabra, en tanto que subsidiario del central, es el máximo responsable del cierre por sus políticas corruptas. Por eso, proponen nuevas elecciones: en caso de ganarlas, prometen reabrir RTVV.

Por su parte, los trabajadores no se centran tanto en buscar responsables (tal y como establecíamos en nuestra hipótesis de partida) como en defender su buen oficio en la Corporación. Son los actores que más hincapié hacen en que el cierre de RTVV significa la pérdida de un espacio cultural propio. Ante esta situación, animan especialmente a los ciudadanos a movilizarse de manera activa. Los medios de comunicación son los actores que incluyen una mayor diversidad de voces sobre el proceso de cierre. Son los que más claramente aluden a una posible censura 
existente en RTVV y los que más abiertamente hablan del conflicto entre periodistas y gobierno valenciano. Finalmente, la participación de las universidades en este debate es anecdótica.

Es necesario destacar el papel activo del colectivo más afectado por la medida; esto es, los periodistas. Ellos han sido, sobre todo, los que han introducido nuevos temas, han valorado de manera más intensa el cierre, han apuntado posibles soluciones y han animado a los ciudadanos a protestar y movilizarse. La oposición ha sido la que más han usado la plataforma para atribuir responsabilidades frente al papel más institucional de los medios, que se han limitado a informar, y de los centros de enseñanza, que no han ido más allá de la propia actualidad universitaria. Una vez más, pues, se apunta al uso de la plataforma de microblogging como mecanismo ideal para difundir contenidos de resistencia al poder, sensibilizar a los ciudadanos y movilizarlos.

El análisis del discurso sobre cierre de RTVV en Twitter por parte de sus protagonistas ha permitido constatar que la plataforma permite a aquellos colectivos más directamente afectados introducir nuevos temas y orientar el debate hacia la condena de lo sucedido, la rendición de cuentas y la propuesta de soluciones. Un uso compatible con la cobertura en tiempo real de lo que estaba sucediendo compartido además con el resto de actores implicados. El uso de los hashtags, en todo caso, refuerza el mensaje principal con el fin de hacerlo más visible: que RTVV no puede cerrarse y que algún día volverá.

\section{Referencias bibliográficas}

Albornoz, L.A., y Cañedo Ramos, A. (2015). La reflexión académica sobre la televisión autonómica en España entre la reivindicación de la diversidad y la denuncia por manipulación política. En J. Marzal Felici (coord), Las televisiones públicas autonómicas del siglo XXI: nuevos escenarios tras el cierre de RTVV (pp. 29-53). Valencia: Aldea Global.

Alzamora, G., y Braga, C (2014). Las redes sociales, armas de protesta. Twitter y Facebook en las protestas de movimientos sociales en España y Brasil. En C. Ferré Pavia (ed.), El uso de las redes sociales: ciudadanía, política y comunicación La investigación en España y Brasil (pp.16-28). Bellaterra: Institut de la Comunicació.

Ardévol-Abreu, A. (2015). Framing o teoría del encuadre en comunicación: Orígenes, desarrollo y panorama actual en España. Revista Latina de Comunicación Social, 70, 423-450. doi: 10.4185/RLCS-2015-1053

Asur, S., Huberman, B., Szabo, G., y Wang, C. (2011). Trends in social media: persistence and decay. $5^{\text {th }}$ International AAAI Conference on Weblogs and Social Media. Recuperado de http://www.hpl.hp.com/research/scl/papers/trends/trendsweb.pdf 
Bennet, W. L., y Segerberg, A., (2012). The logic of connective action. Digital media and the personalization of contentious polítics. Information. Comunicación \& Society, 15(5), 739-768.

Benford, R. D., y Snow, D. A. (2000). Framing Processes and Social Movements: An Overview and Assessment, Annual Review of Sociology, 26, 611-639.

Benson, R., y Powers, M., (2011). Public Media and Political Independence: Lessons for the Future of Journalism from Around the World. Recuperado de: http:// www.savethenews.org/files/public-media-and-political-independence.pdf

Berná Sicilia, C., Pérez Diaz, P. L. y Arroyas Langa, E. (2014). Twitter y la prensa: dos relatos sobre la "Ley Wert". Historia y Comunicación Social, 19, núm. Especial Febrero, 689-704.

Bernal Triviño, A. I., (2015). Tecnología, redes sociales, política y periodismo: ¿Pluralidad informativa o efecto bumerán? Cuadernos.info, 36, 191-205. doi: 10.7764/cdi.36.647

Bruns, A., y Burguess, J., (2012). Researching news discussion on Twitter. Journalism Studies, 13(5-6), 801-814.

Cárcar Benito, J. E. (2015). Las redes y los movimientos sociales ¿una acción colectiva o marketing viral? Icono 14, 13, 125-150.

Carrera Álvarez, P., Sainz de Baranda Andújar, C., Herrero Curiel, E., y Limón Serrano, N., (2012). Journalism and Social Media: How Spanish Journalists Are Using Twitter. Estudios sobre el mensaje periodístico,18(1), 31-53.

Carter, M.; Tsagkias, M., y Weerkamp, W., (2011).Twitter hashtags: Joint Translation and Clustering. En Proceedings of the ACM WebSci'11, June 14-17 2011, pp. 1-3. Koblenz: Germany

Casero-Ripollés, A. y Feenstra, R.A. (2012). The 15-M Movement and the new media: A case study of how new themes were introduced into Spanish political discourse. MIA. Media International Australia, 144, 68-76.

Casero-Ripollés, A., Feenstra, A. y Tormey, S. (2016). Old and New Media Logics in an Electoral Campaign The Case of Podemos and the Two-Way Street Mediatization of Politics. The International Journal of Press/Politics, 1-20. doi: $10.1177 / 1940161216645340$.

Castells, M. (2009). Communication Power. Oxford: Oxford University Press.

Castells, M (2012). Redes de indignación y esperanza: los movimientos sociales en la era de internet. Madrid: Alianza Editorial.

Castells, M. (2014). El poder de las redes. La Vanguardia, dossier 50, pp. 6-13.

Chadwick, A. (2011). The Political Information Cycle in a Hybrid News System: The British Prime Minister in the 'Bullygate' Affair. International Journal of Press/Politics, 16 (1), 3-29.

Dahlberg-Grunberg, M., y Lindgren, S. (2014). Translocal Frame Extension in a Networked Protest: situating the \#IdleNoMore. IC-Revista Científica de Comunicación, 11, 49-79.

De Fleur, M., y Ball- Rockeach, S. J. (1993). Teorías de la comunicación de masas. Barcelona: Paidós.

Della Porta, D. (2011). Communication in movement: Social movements as agents of participatory democracy. Information Communication and Society, 14(6), 800-819. 
Entman, Robert M. (1993). Framing: Toward Clarification of a fractured paradigm. Journal of Communication, 43(4), 51-58.

Fernández Lombao, T., y Campos Freire, F. (2013). La Responsabilidad Social Corporativa en las radio-televisiones públicas de Europa. Cuadernos.info, 33, 145-157.

Flores Vivar, J. (2009). Nuevos modelos de comunicación, perfiles y tendencias en las redes sociales. Comunicar, 17(33), 73-81.

Gans, H. (1980). Deciding what's news. New York: Vintage.

García Santamaría, J. V., y Pérez Serrano, M. J. (2015). Telemadrid: crónica de un déja vu en el contexto de las televisiones públicas autonómicas. En: J. Marzal Felici (coord), Las televisiones públicas autonómicas del siglo XXI: nuevos escenarios tras el cierre de RTVV (pp. 107-126). Valencia: Aldea Global.

Gitlin, T. (1980). The whole world is watching. Mass media in the making and unmaking of the New Left. Berkeley: University of California Press.

Goffman, E. (1974). Frame Analysis. An Essay on the Organization of Experience. Boston: Northeastern University Press.

González-Molina, S. y Ramos del Cano, F. (2015). El cierre de RTVV en las redes sociales: el debate en Twitter a través de sus protagonistas. En J. Marzal Felici (coord), Las televisiones públicas autonómicas del siglo XXI: nuevos escenarios tras el cierre de RTVV (pp. 257-274). Valencia: Aldea Global.

Grossi, G. (1985). Professionalità giornalistica e costruzione sociale della realtà. Problemi dell'informazione, 10(3), 375-388.

Haptom, K., Rainie, L., Lu, W., Dwyer, M., Shin, I., y Purcell, K. (2014). Social media and the spiral of silence. Washington, D.C.: Pew Research. Recuperado de http://www.pewinternet.org/2014/08/26/social-media-and-the-spiral-of-silence/.

Hermida, A. (2010). Twittering the news: The emergence of ambient journalism. Journalism Practice, 4(3), 297-308.

Huberman, B., Romero, D. M., y Wu, F. (2009). Social networks that matter: Twitter under the microscope. First Monday, 14(1).

Instituto Reuters (2016). Digital News Report 2016. Recuperado de http://reutersinstitute.politics.ox.ac.uk/sites/default/files/Digital-News-Report-2016.pdf

Lazarsfeld, P. F., Bernard Berelson, B., y Gaudet, H. (1944). The Peode's Choice. New York: Duell, Sloan \& Pearce.

López Meri, A. (2015). El impacto de Twitter en el periodismo: un estado de la cuestión. Revista de la Asociación Española de Investigación de la Comunicación, 2(4), 34-41.

McNair, B. (2006). Cultural chaos. Journalism, news and power in a globalised world. London: Routledge.

Micó, J. L y Casero-Ripollés, A. (2013): Political activism online: organization and media relations in the case of $15 \mathrm{M}$ in Spain. Information, Communication \& Society, 17(7), 858-871.

Miguel de Bustos, J. C., y Casado, M. A. (2012). Televisiones autonómicas: Evolución y crisis del modelo público de proximidad. Barcelona: Gedisa.

Miguel de Bustos, J. C., y Casado, M. A. (2015). La crisis de la radiotelevisión pública autonómica En J. Marzal Felici, J. Izquierdo Castillo, y A. Casero-Ripollés (coords): La crisis de la televisión pública: el caso de RTVV y los retos de una nueva gobernanza (pp. 61-77). Valencia: Aldea Global. 
Naaman, M., Becker, H. y Gravano, L. (2011). Hip and trendy: Characterizing emerging trends on Twitter. Journal of the American Society for Information Science and Technology, 62(5), 902-918.

Palau-Sampio, D., Carratalá, A., y Valero-Ordaz, L. (2017). Mediatización y encuadres de campaña. Análisis comparado de la información de partidos y medios en las elecciones generales de 2015 en España. El profesional de la información, 26(4), 602-610.

Poell, T. y Borra, E. (2012). Twitter, YouTube, and Flickr as Platforms of Alternative Journalism. The Social Media Account of the 2010 Toronto G20 Protests. Journalism, 13(6), 695-713.

Quintana, Y., y Tascón, M. (2015). De los ‘memes' a la construcción de una nueva narrativa. Revista de Pensamiento sobre Comunicación, Tecnología y Sociedad, 100, 52-54.

Rojo, R. (2012). Existe alguna relación entre la audiencia televisiva y la cantidad de tweets sobre un programa: el caso de Mundos Opuestos. (Tesis Doctoral). Santiago de Chile. Facultad de Economía y Negocios. Universidad de Chile.

Sádaba, T., Rodríguez-Virgili, J. y Bartolomé, M. (2012). Propuesta de sistematización de la teoría del framing para el estudio y praxis de la comunicación política. Observatorio (OBS*) Journal, 6(2), 109-126.

Segerberg, A. y Lance Bennett, W. (2011). Social media and the organization of collective action: Using Twitter to explore the ecologies of two climate change protests. The Communication Review, 14(3), 197-215.

Sherman, A., Arriaga, A., y Valenzuela, S. (2013). La protesta en la era de las redes sociales: el caso chileno. En A. Arriaga y P. Navia (eds.): Medios de comunicación y democracia en Chile (pp. 179-198). Santiago de Chile: Ediciones Universidad Diego Portales.

Soler Campillo, M., y Marzal Felici, J. (2015). La relevancia estratégica de RTVV en el sistema comunicativo valenciano y para el desarrollo económico, social y cultural de la Comunidad Valenciana. En J. Marzal Felici, J. Izquierdo Castillo, y A. Casero-Ripollés (coords): La crisis de la televisión pública: el caso de RTVVy los retos de una nueva gobernanza (pp.113-143). Valencia: Aldea Global.

Toret, J. (coord.) (2013). Tecnopolítica: la potencia de las multitudes conectadas. El sistema red 15M, un nuevo paradigma de la política distribuida. Barcelona: UOC-IN3.

Tuchman, G. (1983). La producción de la noticia. Estudio sobre la construcción de la realidad. Barcelona: Gustavo Gili.

Valenzuela, S., Park, N., y Kee, K. F. (2009). Is There Social Capital in a Social Network Site?: Facebook Use and College Students' Life Satisfaction, Trust, and Participation. Journal of Computer-Mediated Communication, 14(4), 875-901.

Valera-Ordaz, L. (2016). El sesgo mediocéntrico del framing en España: una revisión crítica de la aplicación de la teoría del encuadre en los estudios de comunicación, Zer, 21(41), 13-31.

Valera-Ordaz, L., y López (2014). Agendas y marcos en las webs del PP y PSOE en la cibercampaña de 2011, Revista Latina de Comunicación Social, 69, 41-66.

Vallespín, F. (2011). Redes sociales y democracia: ¿un cambio cualitativo? Recuperado de https://telos.fundaciontelefonica.com/seccion=1268\&idioma=es_ ES\&id=2011102410060001\&activo=6.do 
Van Laer, J. y Van Aelst, P. (2010). Internet and Social Movement Action Repertoires. Opportunities and limitations. Information, Communication \& Society, 13(8), 1-26.

Vicente Mariño, M., y López-Rabadán, P. (2009). Resultados actuales de la investigación sobre framing: sólido avance internacional y arranque de la especialidad en España. Zer, 14 (26), 13-34.

Wu, S., Hofman, J., Mason W., y Watts, D. (2011). Who says what to whom on twitter. En Proceedings of the 20th international conference on World wide web, ACM, 705-714. 